\title{
Expression of long-chain fatty acid receptor GPR40 is associated with cancer progression in colorectal cancer: A retrospective study
}

\author{
CHIE NAKASHIMA $^{1}$, KISHI SHINGO ${ }^{1}$, RINA FUJIWARA-TANI ${ }^{1}$, YI LUO ${ }^{1,2}$, ISAO KAWAHARA ${ }^{1}$, \\ KEI GOTO $^{1}$, TAKAMITSU SASAKI ${ }^{1}$, KIYOMU FUJII ${ }^{1}$, HITOSHI OHMORI ${ }^{1}$ and HIROKI KUNIYASU ${ }^{1}$ \\ ${ }^{1}$ Department of Molecular Pathology, Nara Medical University, Kashihara, Nara 634-8521, Japan; \\ ${ }^{2}$ Key Laboratory of Neuroregeneration of Jiangsu and Ministry of Education, \\ Co-innovation Center of Neuroregeneration, Nantong University, Nantong, Jiangsu 226001, P.R. China
}

Received November 6, 2017; Accepted March 16, 2018

DOI: $10.3892 / 01.2018 .8383$

\begin{abstract}
An increased risk of colorectal cancer (CRC) is associated with a western style diet, particularly hyperlipidemia. The expression of G-protein coupled receptor 40 (GPR40), a membrane-bound receptor for long-chain fatty acids (LCFAs), was examined in 36 cases of subserosal-invading CRC and compared with clinicopathological parameters as well as triglyceride (TG) and low-density lipoprotein (LDL) levels in the blood. All patients with CRC expressed GPR40, which was positively associated with blood TG levels $(\mathrm{P}<0.0001)$ but not with blood LDL levels. GPR40 expression was positively associated with nodal metastasis, distant metastasis (particularly to the liver), stage and poor prognosis. Patients with high GPR40 expression and high TG levels had comparatively worse survival outcomes compared with patients with low GPR40 expression and low TG levels. The results of the present study suggest that activation of GPR40 may be associated with the progression and prognosis of CRCs. High levels of GPR40 and/or concurrent high levels of GPR40 and TG may be a risk for CRC progression.
\end{abstract}

\section{Introduction}

Colorectal cancer (CRC) is the third leading cause of cancer-related death in Japan (1). The number of patients with

Correspondence to: Professor Hiroki Kuniyasu, Department of Molecular Pathology, Nara Medical University, 840 Shijo-cho, Kashihara, Nara 634-8521, Japan

E-mail: cooninh@zb4.so-net.ne.jp

Abbreviations: CRC, colorectal cancer; LCFA, long-chain fatty acid; GPCR or GPR, G-protein coupled receptor; TG, triglyceride; LDL, low-density lipoprotein; EA, elaidic acid; RAGE, receptor for advanced glycation end-products; EMT, epithelial-mesenchymal transition; EGFR, epidermal growth factor receptor; AGE, advanced glycation end-products

Key words: GPR40, long-chain fatty acid, hyperlipidemia, metastasis
$\mathrm{CRC}$, and the number CRC-related mortalities have increased in Japan, probably as a result of the increase in popularity of a western lifestyle $(2,3)$. Some dietary ingredients such as fatty acids have been proposed to increase the risk of CRC (4).

Some long-chain fatty acids (LCFAs) possess pro-tumoral activity. Linoleic acid enhances colon carcinogenesis and metastasis by activating the receptor for advanced glycation end-products (RAGE) and high mobility group box-1 in the azoxymethan-induced rat colon cancer model $(5,6)$. Linoleic acid also inhibits proliferation of cancer cells and induces quiescence (7,8). Elaidic acid (EA), a trans-fatty acid, enhances metastatic potential in colon cancer cells by induction of stemness and promoting epithelial mesenchymal transition (EMT) $(9,10)$.

The LCFAs bind to, and mediate functional effects through, specific membrane-bound receptors: the G-protein coupled receptor 40 (GPR40) and GPR120 receptors (11). The GPR40 is expressed in the beta cells of the pancreatic islets to affect insulin secretion (12), and binds to various LCFAs. This receptor exhibits ligand-biased signaling, and binding to oleic acid causes it to couple to Gq type of G-protein, stimulating calcium ion influx and inositol phosphate synthesis (13). In contrast, EA-induced GPR40-signaling transactivates epidermal growth factor receptor (EGFR) and c-SRC signaling (10). Activation of GPR40 is essential for multifunctional effects of LCFAs.

In the body, LCFAs are supplied as part of triglycerides (TGs) and reach various tissues as well as tumor cells from circulating blood (14). Thus, evaluation of GPR40 expression status and plasma TG levels in CRC patients would be useful in elucidating the role of LCFAs in CRC. In the present study, we examined GPR40 expression and plasma TG levels in $36 \mathrm{CRC}$ patients and sought to understand if fatty acids affect CRC progression and survival outcomes.

\section{Patients and methods}

Patients. We randomly selected 36 patients with pT3-CRC diagnosed pathologically in the Department of Molecular Pathology, Nara Medical University (Kashihara, Japan) from 2012 to 2015. All cases were treated with curative resection, without a history of diabetes mellitus. Written informed 
consent was not required as any identifying information was removed from the samples prior to analysis, to ensure strict privacy protection (unlinkable anonymization). All procedures were performed in accordance with the Ethical Guidelines for Human Genome/Gene Research enacted by the Japanese Government, which was approved by the Ethics Committee of the Nara Medical University (approval no. 937).

Immunohistochemistry. Consecutive 4- $\mu \mathrm{m}$ sections of resected tissue were immunohistochemically stained using the immunoperoxidase technique described previously (15). Anti-GPR40 antibody (Abnova, Walnut, CA, USA) was used at a concentration of $0.2 \mu \mathrm{g} / \mathrm{ml}$. Secondary antibodies (Medical and Biological Laboratories, Nagoya, Japan) were used at a concentration of $0.2 \mu \mathrm{g} / \mathrm{ml}$. Tissue sections were color-developed with diamine benzidine hydrochloride (DAKO, Glastrup, Denmark), and counterstained with Meyer's hematoxylin (Sigma Chemical Co., St. Louis, MO, USA). A GPR40 expression score was calculated by multiplying the staining strength score (0-2) with the staining area (0-5), which yielded scores ranging from 0 to 10 .

Statistical analysis. Statistical analyses of experimental data were carried out using the Spearman $r$ test, analysis of variance (ANOVA), and the two-tailed chi-squared test (InStat; Graphpad Software Inc., La Jolla, CA, USA). A Bonferoni test was performed after ANOVA as a post hoc test. Survival analysis was performed using the Kaplan-Meier method along with the log-rank test. Univariate and multivariate analyses were performed using the log-rank trend test and the Cox's hazard model, respectively (SPSS Statistics, IBM Japan, Tokyo, Japan). Statistical significance was defined as a two-sided P-value $<0.05$.

\section{Results}

Expression of GPR40. In 36 CRCs with sub-serosal or sub-adventitial layer invasion (pT3), (patients without a history of diabetes mellitus), GPR40 expression was examined immunohistochemically (Fig. 1). Eighteen cases displayed relatively high GPR40 expression, and were attributed a 'GPR40-high' status, while 18 other cases with relatively low GPR40 expression were given a 'GPR40-low' status (Table I). In GPR40-high samples, GPR40 immunoreactivity was identified in the plasma membrane (Fig. 1A). In contrast, in GPR40-low samples, a signal of weak intensity was observed in relatively few cells.

Interestingly, a 'GPR40-high' status was associated significantly with lymph node metastasis, liver metastasis, and stage, but not with histological differentiation.

GPR40 expression and blood TG levels. Next, we compared GPR40 expression with TG or low-density lipoprotein (LDL) levels (Fig. 2). A 'GPR40-high' status was associated with higher blood TG levels, but not with blood low density lipoprotein (LDL) levels. Moreover, TG levels were associated with stage, whereas LDL levels were not associated with stage (Table II).

The samples were divided into the two groups by GPR40 expression status and blood TG levels. The first group consisted of samples with both high GPR40 status as well as high TG

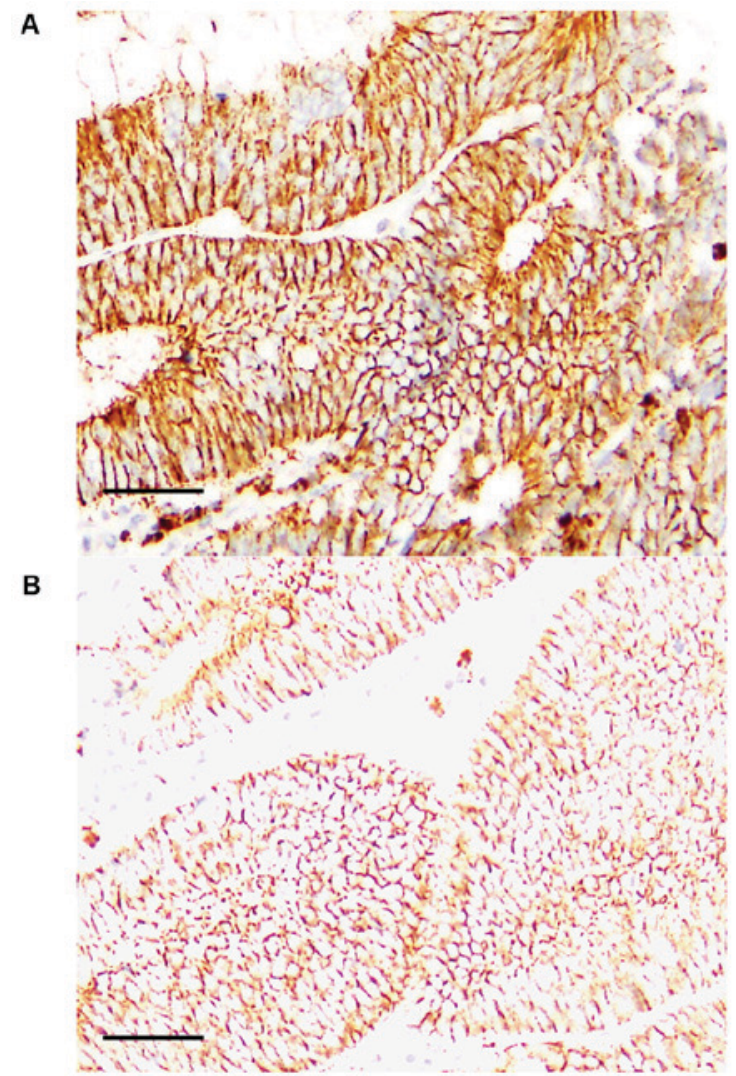

Figure 1. Expression of GPR40 in colorectal cancer. GPR40 expression was examined by immunohistochemistry. (A) A case of tub2, stage IV, pT3, pN2, pM1 (liver). GPR40 expression was 10. (B) A case of tub1, stage II, pT3, pN0, pM0. GPR40 expression was 0.5 . Scale bar, $100 \mu \mathrm{m}$. Pathological parameters were described according to the tumor, node, metastasis pathological classification system (31). GPR40, G-protein coupled receptor 40.

levels (GPR40:high+TG:high) and the rest of the samples constituted the second group. The two groups were compared with regard to stage, TG levels, and survival outcomes (Table III). The GPR40:high+TG:high group showed more advanced stage and shorter survival periods. Survival analysis showed that the GPR40:high+TG:high group showed worse prognosis compared to that of the other group (Fig. 3).

Table IV shows the results of univariate analysis of clinicopathological parameters. The GPR40 expression status and GPR40+TG levels were the two parameters with the highest statistical significance, followed by liver metastasis (pM). Table V shows results of multivariate analysis; the GPR40 expression status was highly statistically significant, followed by the GPR40+TG parameter, among the clinicopathological parameters analyzed.

\section{Discussion}

The incidence of CRC is on the rise in Japan (1). Life style, especially a western style diet, is implicated as an important causative factor in CRC $(2,3)$. Recent studies revealed a strong relationship between CRC and the metabolic syndrome $(16,17)$ and showed that diabetes mellitus is a risk factor for CRC $(18,19)$. Several factors linking CRC risk with diabetes have been proposed, such as increased expression of insulin-like growth factors; oxidative stress (18); advanced 
Table I. Expression of GPR40 and clinicopathological parameters in $36 \mathrm{pT} 3$ colorectal cancer cases.

\begin{tabular}{lccc}
\hline & \multicolumn{2}{c}{ GPR40 expression ${ }^{\mathrm{a}}$} & \\
\cline { 2 - 3 } Characteristic & Low & High & P-value \\
\hline $\mathrm{n}$ & 18 & 18 & \\
GPR40 levels & 2.7 & 6.7 & $<0.0001$ \\
Age (mean \pm SD) & $68.6 \pm 11.9$ & $69.1 \pm 10.8$ & $\mathrm{NS}$ \\
Sex (M:F) & $8: 10$ & $10: 8$ & $\mathrm{NS}$ \\
Histological grade & & & \\
G1 & 6 & 6 & \\
G2 & 12 & 12 & $\mathrm{NS}$ \\
pT3 & 18 & 18 & \\
pN 0 & 15 & 3 & \\
pN 1-2 & 3 & 15 & 0.0002 \\
pM 0 & 18 & 12 & \\
pM 1 & 0 & 6 & 0.0191 \\
Stage & & & \\
II & & 3 & \\
III & 15 & 9 & \\
IV & 3 & 6 & 0.0002 \\
TG (mean \pm SD) & $121.9 \pm 23.9$ & $190.8 \pm 65.7$ & 0.0002 \\
LDL (mean \pm SD) & $124.2 \pm 15.7$ & $126.8 \pm 25.6$ & NS \\
\hline
\end{tabular}

${ }^{a}$ GPR40 expression was calculated as the staining strength score (0-2) multiplied by the staining area (0-5), which yielded scores

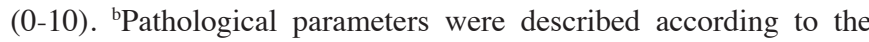
tumor, node, metastasis pathological classification system (32). G1, well differentiated; G2, moderately differentiated; pT3, tumor invades sub-serosal or sub-adventitial layer; $\mathrm{pN} 1-2$, metastasis in regional lymph nodes; pM1, metastasis confined to the liver. GPR40, G-protein coupled receptor 40; TG, blood triglyceride ( $\mathrm{mg} / \mathrm{ml})$; LDL, low-density lipoprotein $(\mathrm{mg} / \mathrm{ml})$; NS, not significant; M, male; F, female; SD, standard deviation.

glycation end-products (AGE; which may enhance malignant potential of colorectal cells) $(5,20)$; and activation of the renin-angiotensin system (21). Hypertriglyceridemia or hyper low-density lipoproteinemia is associated with colonic adenomas, though the risk contribution of these factors is controversial $(22,23)$.

The membrane-bound receptors of LCFA (which are components of TGs) are G-protein coupled receptors (GPCRs), of which GPR40 and GPR120 are well known $(11,24)$. However, the differential biological activities of fatty acids cannot be fully explained by the activity of these receptors alone. To fully explore the spectrum of fatty acid functionality, the activities of cytoplasmic receptors, bioactive metabolites of fatty acids, and those of fatty acids integrated into the plasma membrane need to be studied $(7,8,25)$. In a previous study on trans fatty acids, we showed that EA transactivates EGFR from GPR via c-SRC to increase 'stemness' and induce the epidermal-to-mesenchymal transition in cancer cells (10). Oleic acid also transactivates EGFR via c-SRC signaling (26). The diverse activities of fatty acids might thus be a result of activation of multiple GPCR-dependent and GPCR-independent pathways.
Table II. Association between stage and TG or LDL in patients with colorectal cancer.

\begin{tabular}{lrcc}
\hline Parameter & $\mathrm{n}$ & TG & LDL \\
\hline Stage $^{\mathrm{a}}$ & & & \\
II & 18 & $124.8 \pm 36.0$ & $124.8 \pm 15.4$ \\
III & 12 & $177.2 \pm 49.2$ & $122.8 \pm 27.0$ \\
IV & 6 & $209.3 \pm 86.0$ & $133.2 \pm 24.0$ \\
P-value & & 0.0018 & $\mathrm{NS}$
\end{tabular}

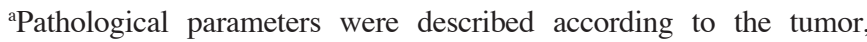
node, metastasis pathological classification system (32). TG, blood triglyceride (mg/ml); LDL, low-density lipoprotein; NS, not significant.
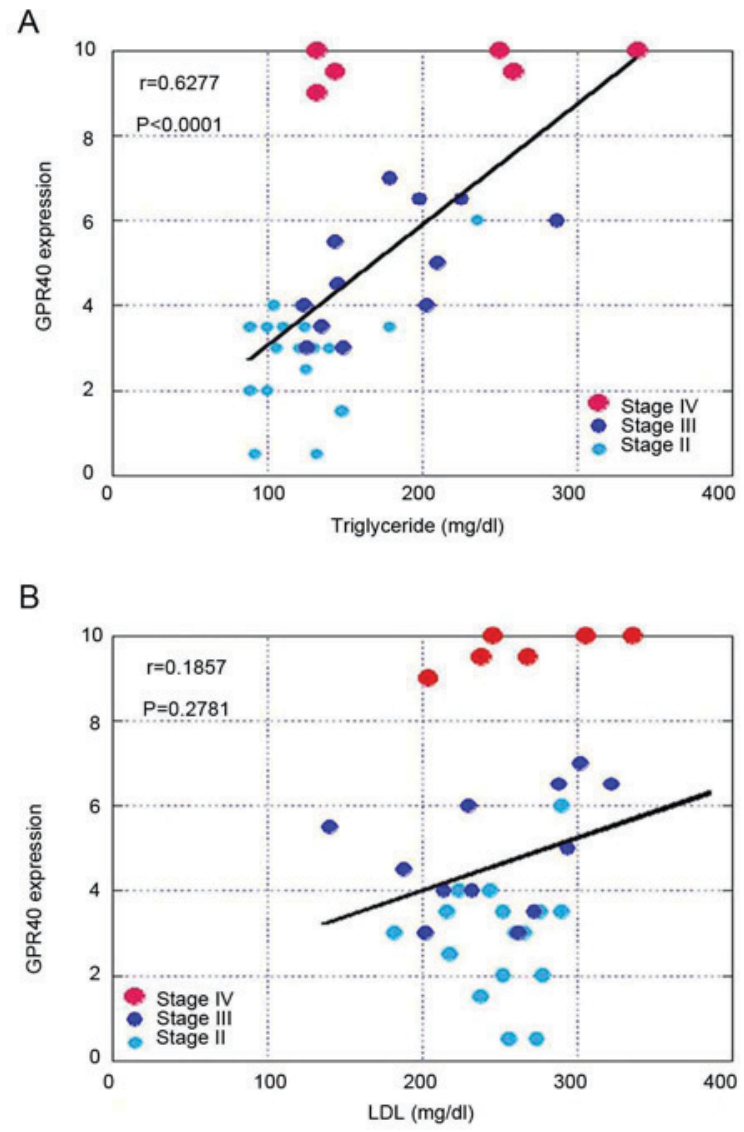

Figure 2. Association between GPR40 expression and TG or low-density LDL levels. (A) Association between GPR40 expression and TG. (B) Association between GPR40 expression and LDL. The $\mathrm{r}$ value and P-value were calculated by Spearman's regression model. GPR40, G-protein coupled receptor 40; TG, triglyceride; LDL, low-density lipoprotein.

In the present study, expression of GPR40 was associated with blood TG levels but not with blood LDL levels. The GPR40 expression was also associated with nodal metastasis, distant metastasis (especially to the liver), stage, and poor prognosis. High GPR40 expression and high TG levels prognosticated worse survival outcomes in our study. These results suggest that GPR40 expression might be linked to CRC progression. Univariate analysis revealed that GPR40 expression status alone showed a stronger association with patient survival than did GPR40:high+TG:high status. This result 
Table III. Comparison of disease stage and survival between patient groups classified by TG and GPR40 expression status.

\begin{tabular}{lccr}
\hline Group & $\begin{array}{c}\text { GPR40:high } \\
\text { +TG:high }\end{array}$ & $\begin{array}{c}\text { Other } \\
\text { cases }\end{array}$ & P-value \\
\hline $\mathrm{n}$ & 13 & 23 & \\
$\begin{array}{l}\text { Stage } \\
\text { II }\end{array}$ & 1 & & \\
III & 8 & 17 & 0.0010 \\
IV & 4 & 2 & \\
TG & $216.9 \pm 58.4$ & $122.1 \pm 21.2$ & $<0.0001$ \\
Survival period & $15.6 \pm 5.4$ & $25.2 \pm 10.4$ & 0.0039 \\
(months) & & &
\end{tabular}

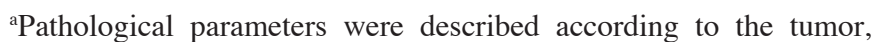
node, metastasis pathological classification system (32). GPR40, G-protein coupled receptor 40; TG, blood triglyceride ( $\mathrm{mg} / \mathrm{ml})$.

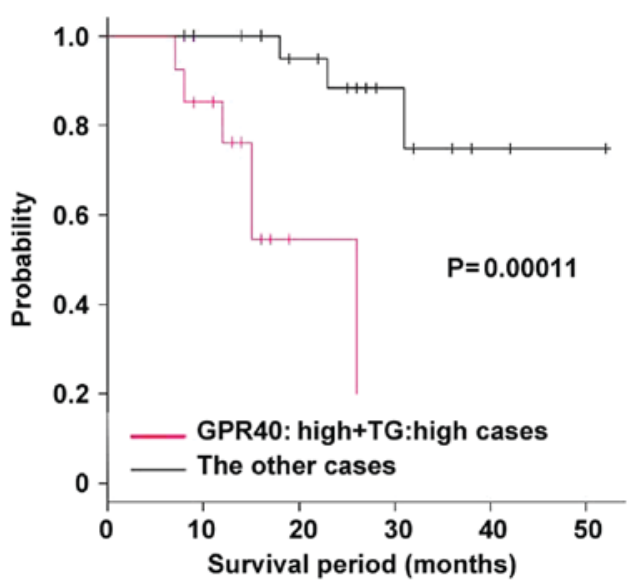

Figure 3. Overall survival of patients with colorectal cancer. Survival was compared between the two groups; GPR40:high+TG:high cases and the rest of the cases (the other cases). Survival analysis was performed by using the Kaplan-Meier method along with the log-rank test. TG, triglyceride; GPR40, G-protein coupled receptor 40 .

suggests that heterogeneity in LCFA content of TGs might be a confounding factor and may be masking the significant influence of GPR40 expression in the case of patients with a GPR40:high+TG:high status.

Our recent reports show different effect of some long chain fatty acids. Linoleic acid, EA and oleic acid are examined on their effects to cancer cells. Linoleic acid provides increased stemness with dormancy (7). EA enhanced metastability by increased proliferative stemness (10). In. contrast, oleic acid does not increase metastability (10). Univariate and multivariate analyses showed GPR40 is more significant than GPR40+TG. As described above, different fatty acid provides different effect. TGs is a mixture of many fatty acids, which might cancel the effects each other. Then it should be needed clarifying content of each fatty acid. It does not seem that GPR40 and TG levels in stage IV cases. In stage IV cases, other pro-metastatic factors, such as growth factors might cause more relevant contributions to metastasis.
Table IV. Univariate analyses of clinicopathologic parameters.

\begin{tabular}{lrr}
\hline Parameter & Chi-squared & P-value \\
\hline Stage $^{\mathrm{a}}$ & & \\
II, III, IV & 11.3000 & 0.000760 \\
pN (0,1-2) & 14.0000 & 0.000130 \\
pM (0,1) & 20.2000 & 0.000007 \\
TG (high, low) & 7.8700 & 0.005000 \\
GPR40 & 18.7000 & 0.000015 \\
GPR40+TG & 13.3000 & 0.000270 \\
(GPR40:high+TG:high, others) & & \\
\hline
\end{tabular}

apathological parameters were described according to the tumor, node, metastasis pathological classification system (32); pN1-2, metastasis in regional lymph nodes; pM1, metastasis confined to the liver. GPR40, G-protein coupled receptor 40; TG, blood triglyceride $(\mathrm{mg} / \mathrm{ml})$.

Table V. Multivariate analyses of clinicopathologic parameters.

\begin{tabular}{lcccc}
\hline & $\begin{array}{c}\text { Hazard } \\
\text { ratio }\end{array}$ & $\begin{array}{c}\text { Lower } \\
95 \%\end{array}$ & $\begin{array}{c}\text { Upper } \\
95 \%\end{array}$ & P-value \\
\hline GPR40 & 4.787 & 1.453 & 15.77 & 0.01004 \\
GPR40+TG & 2.649 & 1.123 & 6.249 & 0.02613 \\
(GPR40:high+ $_{\text {TG: high, others) }}$ & & & & \\
Stage (II, III, IV) & 0.0627 & 0.004189 & 0.9387 & 0.04488 \\
\hline
\end{tabular}

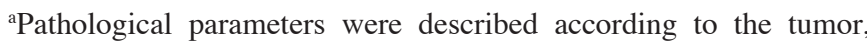
node, metastasis pathological classification system (32); pN1-2, metastasis in regional lymph nodes; pM1, metastasis confined to the liver. GPR40, G-protein coupled receptor 40; TG, blood triglyceride $(\mathrm{mg} / \mathrm{ml})$.

Our previous studies show that linoleic acid (an n-6 LCFA) and EA (a trans fatty acid), enhance colon carcinogenesis or metastability of CRC $(5,6,9,10)$, whereas docosahexaenoic acid (DHA) and eicosapentaenoic acid (EPA) (n-3 LCFAs) suppress colon carcinogenesis and enhance efficacy of chemotherapy regimens (27). The n-3 LCFAs also activate the hippo system via GPR40 and GPR120 to inhibit proliferation of CRC (28). Although blood TG level is widely utilized as a predictive clinical parameter, the identity of the fatty acids that constitute the TGs might be an important factor that determines the relationship between TGs and cancer risk.

Metabolic syndrome is a collection of risk factors, such as hypertension, higherglycemia, hyperlipidemia, and visceral fat based on insulin resistance (29). Diets of patients with the metabolic syndrome are reported to contain fat with a high n-6/n-3 fatty acid ratio (30) as well as a high content of trans fatty acids (31). From these findings, metabolic syndrome-associated hypertriglyceridemia might be suggested to be pro-tumoral for CRC. In the cases examined here, the blood TG level was associated with survival outcomes of the patients, which suggests that 
hypertriglyceridemia needs to be closely examined with reference to CRC progression.

The association between GPR40 with blood TG levels may imply that TGs induce GPR40 expression in CRC. In contrast, LCFAs suppress GPR40 expression in pancreatic islet beta cells to adversely affect diabetes (12). The mechanism of regulation of GPR40 expression has not been fully elucidated, though LCFAs have been implicated. The signaling pathways leading to GPR40 overexpression and the effect of dyslipidemia on such signaling need to be examined in future studies.

\section{Acknowledgements}

The authors would like to thank Ms. Tomomi Masutani for assistance with the preparation of this manuscript.

\section{Funding}

The present study was supported by MEXT KAKENHI (grant nos. 16H05164, 17K15648, 17K19923 and 16K19087).

\section{Availability of data and materials}

All data generated or analyzed during the present study are included in this published article.

\section{Authors' contributions}

HK designed the study. CN, KS, RFT and YL collected and analyzed the data. IK, KG, TS, HO and KF analyzed the data. $\mathrm{CN}$ and $\mathrm{HO}$ prepared and had final approval of the manuscript.

\section{Ethics approval and consent to participate}

The present study was approved by the Ethics Committee of the Nara Medical University (approval no. 937).

\section{Consent for publication}

Not applicable.

\section{Competing interests}

The authors confirm that they have no competing interests.

\section{References}

1. Wakao F, Nishimoto H, Kataonoda K, Tsukuma $\mathrm{H}$ and Mikami H (eds): Cancer statistics in Japan, 2013. National Cancer Research Institute, Tokyo, 2013.

2. Kimura Y, Kono S, Toyomura K, Nagano J, Mizoue T, Moore MA, Mibu R, Tanaka M, Kakeji Y, Maehara Y, et al: Meat, fish and fat intake in relation to subsite-specific risk of colorectal cancer: The Fukuoka colorectal cancer study. Cancer Sci 98: 590-597, 2007.

3. Mizoue T, Tanaka K, Tsuji I, Wakai K, Nagata C, Otani T, Inoue $\mathrm{M}$ and Tsugane $\mathrm{S}$; Research Group for the Development and Evaluation of Cancer Prevention Strategies in Japan: Alcohol drinking and colorectal cancer risk: An evaluation based on a systematic review of epidemiologic evidence among the Japanese population. Jpn J Clin Oncol 36: 582-597, 2006.

4. Bultman SJ: Interplay between diet, gut microbiota, epigenetic events, and colorectal cancer. Mol Nutr Food Res 61, 2017.
5. Shimomoto T, Luo Y, Ohmori H, Chihara Y, Fujii K, Sasahira T, Denda A and Kuniyasu H: Advanced glycation end products (AGE) induce the receptor for AGE in the colonic mucosa of azoxymethane-injected Fischer 344 rats fed with a high-linoleic acid and high-glucose diet. J Gastroenterol 47: 1073-1083, 2012

6. Ohmori H, Luo Y, Fujii K, Sasahira T, Shimomoto T, Denda A and Kuniyasu H: Dietary linoleic acid and glucose enhances azoxymethane-induced colon cancer and metastases via the expression of high-mobility group box 1 . Pathobiology 77 : 210-217, 2010.

7. Ohmori H, Sasahira T, Fujii K, Luo Y, Shimomoto T and Kuniyasu H: Linoleic acid-induced growth suppression induces quiescent cancer cell nests in nude mice. Pathobiology 75: 226-232, 2008.

8. Sasaki T, Fujii K, Yoshida K, Shimura H, Sasahira T, Ohmori H and Kuniyasu H: Peritoneal metastasis inhibition by linoleic acid with activation of PPARgamma in human gastrointestinal cancer cells. Virchows Arch 448: 422-427, 2006.

9. Ohmori H, Fujii K, Kadochi Y, Mori S, Nishiguchi Y, Fujiwara R, Kishi S, Sasaki T and Kuniyasu H: Elaidic acid, a trans-fatty acid, enhances the metastasis of colorectal cancer cells. Pathobiology 84: 144-151, 2017.

10. Fujii K, Luo Y, Fujiwara-Tani R, Kishi S, He S, Yang S, Sasaki T, Ohmori H and Kuniyasu H: Pro-metastatic intracellular signaling of the elaidic trans fatty acid. Int $\mathrm{J}$ Oncol 50: 85-92, 2017.

11. Hara T, Kashihara D, Ichimura A, Kimura I, Tsujimoto G and Hirasawa A: Role of free fatty acid receptors in the regulation of energy metabolism. Biochim Biophys Acta 1841: 1292-1300, 2014.

12. Tomita T, Masuzaki H, Iwakura H, Fujikura J, Noguchi M, Tanaka T,Ebihara K, Kawamura J,Komoto I, Kawaguchi Y, et al: Expression of the gene for a membrane-bound fatty acid receptor in the pancreas and islet cell tumours in humans: Evidence for GPR40 expression in pancreatic beta cells and implications for insulin secretion. Diabetologia 49: 962-968, 2006.

13. Mizuta K, Zhang Y, Mizuta F, Hoshijima H, Shiga T, Masaki E and Emala CW Sr: Novel identification of the free fatty acid receptor FFAR1 that promotes contraction in airway smooth muscle. Am J Physiol Lung Cell Mol Physiol 309: L970-L982, 2015.

14. Newsholme EA: The glucose/fatty acid cycle and physical exhaustion. Ciba Found Symp 82: 89-101, 1981.

15. Kuniyasu H, Yasui W, Shinohara H, Yano S, Ellis LM, Wilson MR, Bucana CD, Rikita T, Tahara E and Fidler IJ: Induction of angiogenesis by hyperplastic colonic mucosa adjacent to colon cancer. Am J Pathol 157: 1523-1535, 2000.

16. O'Flanagan $\mathrm{CH}$, Bowers LW and Hursting SD: A weighty problem: Metabolic perturbations and the obesity-cancer link. Horm Mol Biol Clin Investig 23: 47-57, 2015.

17. Schoenberg MH: Physical activity and nutrition in primary and tertiary prevention of colorectal cancer. Visc Med 32: 199-204, 2016.

18. Kasuga M, Ueki K, Tajima N, Noda M, Ohashi K, Noto H, Goto A, Ogawa W, Sakai R, Tsugane S, et al: Report of the Japan diabetes society/Japanese cancer association joint committee on diabetes and cancer. Cancer Sci 104: 965-976, 2013.

19. Shi J, Xiong L, Li J, Cao H, Jiang W, Liu B, Chen X, Liu C, Liu K, Wang $\mathrm{G}$ and Cai K: A linear dose-response relationship between fasting plasma glucose and colorectal cancer risk: Systematic review and meta-analysis. Sci Rep 5: 17591, 2015.

20. Kuniyasu H, Chihara Y and Kondo H: Differential effects between amphoterin and advanced glycation end products on colon cancer cells. Int J Cancer 104: 722-727, 2003.

21. Shimomoto T, Ohmori H, Luo Y, Chihara Y, Denda A, Sasahira T, Tatsumoto N, Fujii K and Kuniyasu H: Diabetes-associated angiotensin activation enhances liver metastasis of colon cancer. Clin Exp Metastasis 29: 915-925, 2012.

22. Yang MH, Rampal S, Sung J, Choi YH, Son HJ, Lee JH, Kim YH, Chang DK, Rhee PL, Kim JJ, et al: The association of serum lipids with colorectal adenomas. Am J Gastroenterol 108: 833-841, 2013.

23. Tian Y, Wang K, Li J, Wang J, Wang Z, Fan Y, Ye Y, Ji G and Li Y: The association between serum lipids and colorectal neoplasm: A systemic review and meta-analysis. Public Health Nutr 18: 3355-3370, 2015. 
24. Yonezawa T, Kurata R, Yoshida K, Murayama MA, Cui X and Hasegawa A: Free fatty acids-sensing G protein-coupled receptors in drug targeting and therapeutics. Curr Med Chem 20: 3855-3871, 2013

25. Kuniyasu H (ed): Linoleic acid. In: Encyclopedia of Cancer. 2nd edition, Springer-Verlag, Berlin, pp1691-1693, 2008.

26. Soto-Guzman A, Robledo T, Lopez-Perez M and Salazar EP: Oleic acid induces ERK1/2 activation and AP-1 DNA binding activity through a mechanism involving Src kinase and EGFR transactivation in breast cancer cells. Mol Cell Endocrinol 294 81-91, 2008.

27. Lee JY, Sim TB, Lee JE and Na HK: Chemopreventive and chemotherapeutic effects of fish oil derived Omega-3 polyunsaturated fatty acids on colon carcinogenesis. Clin Nutr Res 6 : 147-160, 2017.

28. Zhang K, Hu Z, Qi H, Shi Z, Chang Y, Yao Q, Cui H, Zheng L, Han Y, Han X, et al: G-protein-coupled receptors mediate $\omega-3$ PUFAs-inhibited colorectal cancer by activating the Hippo pathway. Oncotarget 7: 58315-58330, 2016.
29. World Health Organization: Definition, diagnosis and classification of diabetes mellitus and its complications: Report of a WHO Consultation. World Health Organization, Geneva, 1999.

30. Simopoulos AP: Essential fatty acids in health and chronic disease. Am J Clin Nutr 70 (3 Suppl): 560S-569S, 1999.

31. Mozaffarian D, Aro A and Willett WC: Health effects of trans-fatty acids: Experimental and observational evidence. Eur J Clin Nutr 63 (Suppl 2): S5-S21, 2009.

32. Brierley JD, Gospodarowicz MK and Wittekind C: TNM Classification of Malignant Tumours. Wiley Blackwell, Oxford, UK, 2017.

cC) $($ This work is licensed under a Creative Commons Attribution-NonCommercial-NoDerivatives 4.0 International (CC BY-NC-ND 4.0) License. 